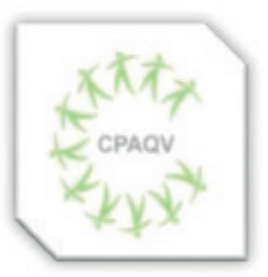

ISSN: 2178-7514

Vol. 14| No. 1| Ano 2022
ARTIGO DE REVISÃO

\section{ATIVIDADE FÍSICA NA PROMOÇÃO DA SAÚDE}

\section{Physical activity in health promotion}

Guanis B. Vilela Junior ${ }^{1}$; Heleise F. R. Oliveira ${ }^{1}$; Adriano A. Pereira ${ }^{1}$; Alexandre F. Carvalho ${ }^{13}$; Anderson Martelli ${ }^{4}$; Anderson S. Carvalho ${ }^{1,6}$; Bráulio N. Lima ${ }^{1}$; Gustavo C. Martins ${ }^{1,9,10,11}$; Klebson S. Almeida ${ }^{1,8}$; Luis F. Silio ${ }^{1}$; Marcelo F. Rodrigues ${ }^{1}$; Mariela S. Maneschy ${ }^{8}$; Pedro P. Abdalla ${ }^{1,5}$; Uebister I. S Guedes ${ }^{7}$; Carlos H. P. Fileni ${ }^{1}$; José Ricardo L. Oliveira ${ }^{1}$; Ricardo P. Passos ${ }^{1,2}$

\title{
RESUMO
}

No mundo contemporâneo, observa-se que desde a infância os indivíduos são influenciados ao uso das tecnologias de maneira excessiva, tornando o sedentarismo cada vez mais presente na vida dos seres humanos. O sedentarismo tem se destacado como um dos fatores negativos para o surgimento de doenças. Para tanto, objetivou-se descrever como a prática da atividade física é capaz de promover a saúde dos indivíduos. Sendo assim, levantou-se a seguinte questão problema: como a prática de atividade física auxilia na promocão da saúde? A metodologia utilizada foi uma pesquisa bibliográfica descritiva, através de base de dados, como: Scięlo; Google Acadêmico, alêm de artigos disponíveis em bibliotecas digitais, sendo utilizadas palavras-chaves, tais como: atividade física; sedentarismo; saúde. Concluindose que a prática da atividade física é relevante para a saúde e bem-estar do ser humano, pois auxilia na prevenção e tratamento de doenças.

Palavras-chave: Atividade Física; Sedentarismo; Saúde.

\begin{abstract}
In the contemporary world, it is observed that since childhood, individuals are influenced to use technologies in an excessive way, making a sedentary lifestyle increasingly present in the lives of human beings. A sedentary lifestyle has been highlighted as one of the negative factors for the emergence of diseases. Therefore, the objective was to describe how the practice of physical activity is capable of promoting the health of individuals. Therefore, the following problem question was raised: how does physical activity help in health promotion? The methodology used was descriptive bibliographic research, through databases, such as: Scielo; Google Scholar, in addition to articles available in digital libraries, using keywords such as: physical activity; sedentary lifestyle; health. Concluding that the practice of physical activity is relevant to the health and well-being of the human being, as it helps in the prevention and treatment of diseases.
\end{abstract}

Keywords: Physical Activity; Sedentary lifestyle; Health.

Autor de correspondência

Ricardo Pablo Passos - rppasso@gmail.com

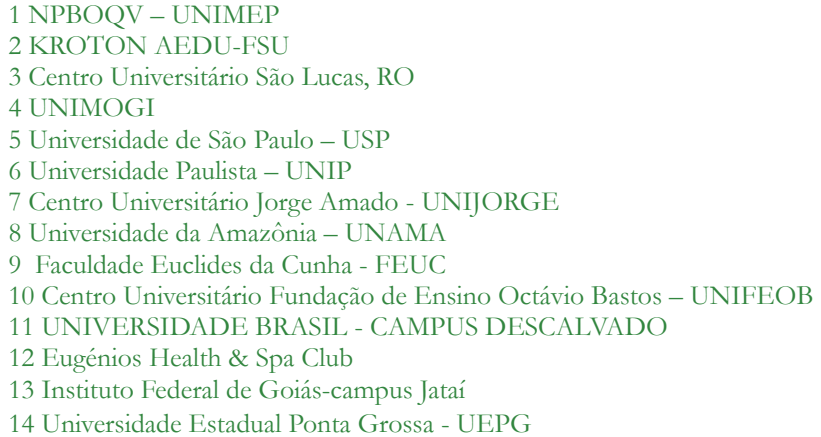

14 Universidade Estadual Ponta Grossa - UEPG 


\section{INTRODUÇÃO}

O sedentarismo está associado a ocorrência dos mais variados tipos de doenças, assim, é fundamental que desde a mais tenra idade seja promovida a prática de atividade física, visando a promoção da saúde.

Todavia, o uso inadequado das tecnologias têm influenciado no aumento do sedentarismo, contribuindo para que o risco de doenças seja elevado, assim como, também que se tenha uma menor qualidade de vida. Nesse contexto, a prática de atividade física demonstra-se como uma forma de promoção á saúde.

Esta pesquisa relaciona-se a possibilidade de destacar a relevância da prática de atividade física na prevenção de doenças e na promoção á saúde, uma vez que, uma gama variada de doenças tem como fator de risco a inatividade física, mostrando-se relevante discutir a respeito dos principais benefícios da prática de atividade física, para todas as faixas etárias

Desta forma, observa-se que desde a infância os indivíduos são influenciados ao uso das tecnologias de maneira excessiva, fazendo com que a inatividade física esteja presente na vida da maior parte dos seres humanos.

Sob esta ótica, definiu-se como objetivo descrever como a prática da atividade física é capaz de promover a saúde dos indivíduos.
Portanto, questionou-se: Como a prática da atividade física é capaz de promover a saúde dos indivíduos?

\section{MÉTODOS}

Realizou-se uma pesquisa bibliográfica descritiva, com o objetivo de identificar sobre a atividade física na promoção da saúde. Para tanto, foram utilizadas as bases de dados; Scielo, Google Acadêmico, além de artigos disponíveis em bibliotecas digitais da área de ciências da saúde por meio de palavras-chaves; atividade física; sedentarismo; saúde.

Como critérios de inclusão somente os artigos completos, publicados em português que referiam-se ao tema da pesquisa. Para os critérios de exclusão, enquadraram-se os trabalhos incompletos e que não versaram sobre o tema.

\section{ATIVIDADE FÍSICA E}

\section{SEDENTARISMO}

Alguns autores ${ }^{1}$ apontam que a inatividade física tem crescido no mundo todo. O estilo de vida contemporâneo tem contribuído para que o sedentarismo e outros males a saúde venham crescendo, visto que a inatividade física é um fator de risco para o desenvolvimento de doenças, sendo assim, cada vez mais relevante que sejam promovidas ações para a inclusão da atividade física, no dia-a-dia do ser humano. 
A vida moderna, em conjunto com os aspectos relacionados ao desenvolvimento das tecnologias, foram responsáveis por um decréscimo na realização das atividades físicas, o que funciona como aumento para o sedentarismo, em conjunto com outros fatores do estilo de vida impactando no crescimento de doenças crônicas degenerativas, como: diabetes; hipertensão arterial; entre outras, estejam presentes em grande parte da população ${ }^{2}$.

Dessa forma, o sedentarismo é um dos fatores de risco para que surjam doenças crônicas, assim como também, responsável pela redução da qualidade de vida e bem-estar dos indivíduos.

O sedentarismo pode ser conceituado como a ausencia da prática de atividade física, com gasto semanal menor do que $1000 \mathrm{Kcal} 3$. Um fator relevante no aumento do sedentarismo foi a mudança na forma de vida do homem, o avanço tecnológico promoveu a inatividade, em conjunto com outros fatores como o uso de tabaco e álcool, e de uma alimentação baseada em alimentos industrializados.

Nesta perpectiva supracitada, as atividades cotidianas do ser humano tem se tornado cada vez menos intensas, ocorrendo também, expressiva mudança nas atividades de lazer que passaram a ser realizadas frente a computadores, celulares e a televisão4. As atividades realizadas ao ar livre também, tem diminuído, considerando a violência urbana e o próprio crescimento da urbanização.
Não obstante, a prática da atividade física de forma regular, associa-se a inúmeros benefícios para a saúde, sobretudo na promoção da prevenção de doenças degenerativas, assim como, também em relação ao sobrepeso e a obesidade, mal este, caracterizado como fator de risco para doenças.

A obesidade é uma doença causada pelo excesso de gordura no organismo, que atinge milhares de pessoas no mundo5, atribuída a uma desproporção entre a ingestão alimentar em excesso e um gasto energético insuficiente.

A obesidade pode ser entendida como uma doença complexa e que apresenta uma multiplicidade de fatores desde emocionais, relacionados ao estilo de vida, a genética e ao meio ambiente em que se vive ${ }^{6}$.

Observa-se, assim, que a prática de atividade física é essencial para que seja possível a melhoria da qualidade de vida do individuo, sendo que as suas vantagens se estendem desde a infância a terceira idade; todavia, em cada fase da vida devem ser analisadas as peculiaridades e individualidades.

Conforme já exposto a obesidade é uma das doenças associadas com a inatividade física, sendo uma de suas consequencias. Alem da obesidade, problemas de saúde como a hipertensão arterial, depressão e a diabetes podem ser prevenidas e tratadas com o auxilio da atividade física ${ }^{1}$

As consequências da inatividade física estão associadas com doenças crônicas, e são 
derivadas de questões relacionadas com o estilo de vida da população, que tem maus hábitos alimentares e um estilo de vida sedentário. As mudanças negativas no estilo de vida são responsáveis por impactar de forma negativa a saúde dos indivíduos, sendo apontada como um fator de aumento dos gastos públicos com a saúde ${ }^{7}$

O autor ainda ressalta que a urbanização provou amplas mudanças no estilo de vida da população, elevando o sedentarismo, modificando a dieta alimentar, fazendo com que se formasse um perfil em que os indivíduos consomem alimentos altamente calóricos e são adeptos da inatividade física, assim, as conseqüências como a obesidade e o sobrepeso, doenças crônicas tem tido a sua prevalência elevada, culminando em uma sociedade cada vez mais adoecida.

Com o objetivo da promoção de mudanças, tem sido promovidas ações de promoção a saúde, a pirâmide alimentar foi modificada abrangendo a prática de atividade física, podendo verificar em [8, F. 1], como uma das bases de uma vida saudável.

\section{PIRÂMIDE DOS ALIMENTOS}

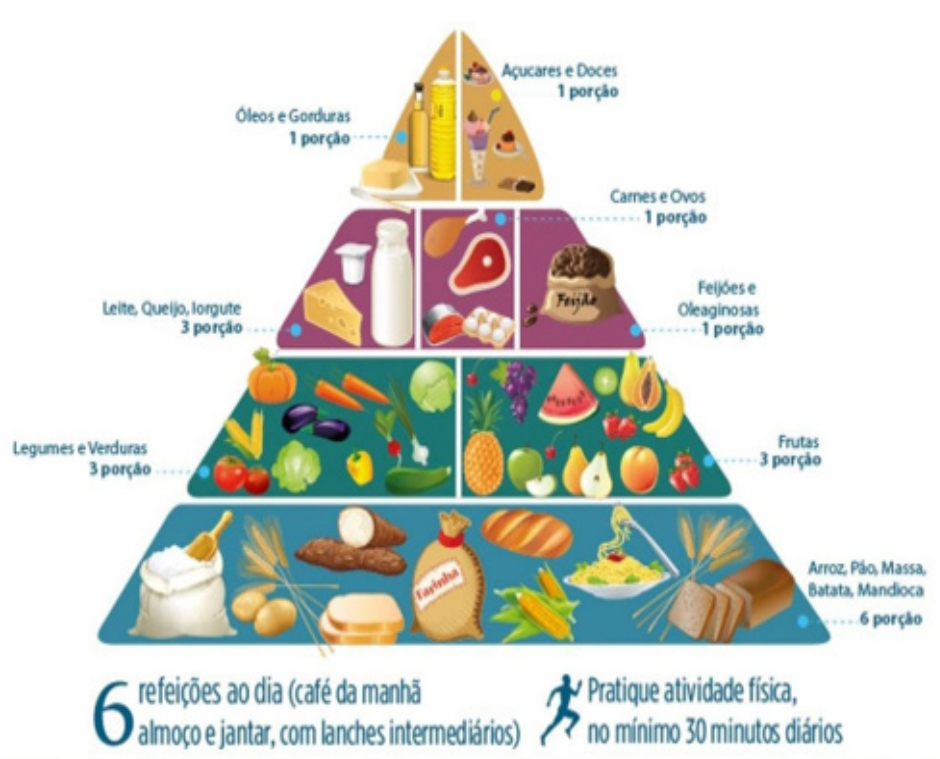

Figura 1- Pirâmide dos alimentos [8]

Assim, destaca-se que a prática da atividade física, por pelo menos trinta minutos por dia, como uma forma de se promover a saúde, estando relacionada com a prevenção de doenças crônicas, altamente prejudiciais a saúde. Desde a infância a atividade física deve ser promovida, ainda nas escolas, sendo estimulada também na vida jovem e adulta e continuada na terceira idade, já que, nessa faixa etária é responsável pela autonomia do idoso. 


\section{APRÁTICADE ATIVIDADE FÍSICA NA PROMOÇÃO A SAÚDE, POR MEIO DA PREVENÇÃO E COMBATE DE DOENÇAS}

A inatividade física, portanto, contribui para que ocorra o sedentarismo e os malefícios a ele relacionados, que comprometem a saúde e o bem-estar do individuo. Esses fatores estão relacionados com as mudanças de hábitos da sociedade moderna, que é propensa a inatividade física e também a uma alimentação inadequada ${ }^{5}$.

A atividade física possibilita que se tenha efeitos positivos em relaçao a saúde, quanto a redução do envelhecimento e na prevenção de doenças crônicas e doenças degenerativas. $\mathrm{O}$ sedentarismo é fator de risco para cada uma dessas doenças, sendo prejudicial a saúde 6 .

Sabe-se que grande parte dos problemas de saúde estão relacionados com o aumento do sobrepeso e da obesidade, estando essa condição relacionada com doenças crônicas, hipertensão, diabetes, entre outras condições maléficas a saúde. As mudanças sociais e econômicas são responsáveis por modificar o estilo de vida da sociedade, que passou por bruscas mudanças em relaçao ao padrão de vida, trazendo impactos negativos para a saúde 7 .

As evoluções tecnológicas também foram um fator primordial quando se fala a respeito de mudança no estilo de vida, elas trazem conforto, mas também fazem com que as pessoas se tornem mais sedentárias Com isso as pessoas que tem um estilo de vida menos ativo fisicamente tem facilitado um aumento do sedentarismo e de seus malefícios para a saúde nas últimas décadas. Desta forma, parece que a saúde das pessoas, a cada dia está deteriorando. Neste contexto, vários distúrbios como ansiedade, depressão, transtornos alimentares e comportamentos compulsivos e obsessivos. Embora o foco de atenção em relação à saúde permanece nas doenças degenerativas associadas com o sobrepeso e a obesidade, há muitas evidências para sugerir que as doenças mentais no mundo estão se tornando a "doença do século XXI", principalmente nos países desenvolvidos e em desenvolvimento (p.6).

A inatividade física é apontada como uma das principais causas responsáveis pelo aumento da mortalidade, sendo responsável por gastos na saúde pública com doenças como as cardio e cerebrovasculares ${ }^{8}$.

O sedentarismo tem se elevado em números alarmantes envolvendo crianças, jovens e adultos. A alimentação também foi prejudicada, com o aumento do consumo de industrializados, alimentos prontos, que empobrecem a alimentação e fazem com que se tenha o consumo de um maior número de calorias, favorecendo o ganho de peso.

O sedentarismo é fator de risco para uma gama de doenças crônicas, conforme já 
citado no capítulo anterior, todavia, é possível relacionar a atividade física com a promoção a saúde, uma vez que, está relacionada a prevenção de doenças crônicas não transmissíveis e também a incapacidade funcional ${ }^{9}$.

A prática de atividade física é considerada como terapêutica desde a antiguidade, locais como a Grécia Antiga e no Oriente, reconhecem a atividade física como necessária para que se tenha saúde, estudos epidemiológicos confirmar a relação entre saúde e atividade física, caracterizando o sedentarismo como fator de risco de varias doenças ${ }^{10}$.

No estudo pioneiro, publicado por Morris et al., comparou-se a prevalência de Doença Arterial Coronariana (DAC) entre carteiros e trabalhadores de escritório do serviço postal de Londres. Foi observado que as atividades ocupacionais com maior gasto energético estavam associadas à menor taxa de morte por Doenças Cardiovasculares (DCV).Outro estudo epidemiológico importante, que revelou forte associação entre atividade física e doenças foi desenvolvido por Paffenbarger et al. Os autores determinaram, (...) ao longo de 16 anos de estudo, declínio progressivo em eventos coronarianos tanto fatais como não fatais, à medida que aumentava o gasto energético diário (menos de $500 \mathrm{kcal} /$ semana a $3500 \mathrm{kcal} /$ semana). Além disso, as taxas de morte foram $25 \%$ a $35 \%$ mais baixas nos indivíduos com gasto energético com atividade física maior ou igual a $2000 \mathrm{kcal} /$ semana, mesmo na presença de outros fatores de risco, como hipertensão, obesidade e fumo. Foi observado também que gastos adicionais tinham pouco ou nenhum efeito na incidência de DCV (COELHO, 2009, p.942).
Dessa forma, evidencia-se que os indivíduos com condicionamento físico adequado estão menos predispostos a doenças crônicas, como dislipidemias, assim, destaca-se que a atividade física funciona como maneira de prevenção precoce de doenças, promovendo a saúde dos indivíduos e fazendo com que se tenha melhorias importantes no organismo ${ }^{11}$.

A realização da atividade física como forma de prevenção de doenças foi promovida a partir do conhecimento de que a prática estaria relacionada com a melhoria do estado geral de saúde, assim como também, quanto ao condicionamento físico geral, composição corporal, entre outros benefícios, sendo recomendada a prática de atividade de forma moderada, pelo menos 30 minutos por dia, cinco dias na semana, para atividade moderadas ${ }^{12}$.

A Organização Mundial da Saúde (OMS) e o Ministério da Saúde (MS) preconizam a prática de atividade física para a prevenção das doenças crônicas, sendo que as recomendações da prática da atividade física são variáveis de acordo com o individuo ${ }^{13}$.

Os tratamentos não medicamentosos das doenças crônicas incluem a atividade física como parte importante, podendo ser inclusas atividades de intensidade moderada, como os exercícios aeróbicos e resistidos. Para os indivíduos que buscam a atividade física para a incapacidade funcional, destaca-se a respeito da relevância de práticas que desenvolvam a flexibilidade e o equilíbrio, por exemplo. 


\section{CONSIDERAÇÕES FINAIS}

A inatividade física está relacionada de forma direta com problemas de saúde, o que pode incluir doenças cardiovasculares, obesidade, problemas metabólicos, entre outras situações maléficas para a saúde. Assim, a prática de atividade física é benéfica a saúde do individuo.

O estilo de vida moderno favorece o uso das tecnologias, traz conforto, mas também promove o sedentarismo e a má alimentação, fatores que em conjunto podem prejudicar a saúde das pessoas.

A atividade física regular é fundamental no combate ao sedentarismo, prevenindo o agravamento de doenças, favorecendo a saúde, o bem-estar, maior qualidade de vida, manutenção do peso corporal, entre outros benefícios que estão atrelados a sua prática regular.

\section{REFERÊNCIAS}

1. AEDO, Ángeles; ÁVILA Hector. Nuevo cuestionario para evaluar la autoeficacia hacia la actividad física em niños. Revista Panamerica Salud Publica. Washington, v.26, n.4, p.324-329, out.2009. Disponível em: . Acesso em: 20 Abr.2021.

2. BRASIL. Ministério da Saúde. RedeNutri Rede de Nutrição do Sistema Único de Saúde. Texto de sistematização: Educação Alimentar e Nutricional. Brasília, 2010.

3. COELHO, Christianne de Faria; BURINI, Roberto Carlos. Atividade física para prevenção e tratamento das doenças crônicas não transmissíveis e da incapacidade funcional. Rev. Nutr., Campinas, v. 22, n. 6, p. 937-946, Dec. 2009 .

4. DUNCAN BB, et al. Mortalidade por doenças crônicas no Brasil: situação em 2009 e tendências de 1991 a 2009. In: Ministério da Saúde (BR). Secretaria de Vigilância em Saúde. Departamento de Análise de Situação em Saúde. Saúde Brasil 2010: uma análise da situação de saúde e de evidências selecionadas de impacto de ações de vigilância em saúde [Internet]. Brasília: Ministério da Saúde; 2011.

5. FARIA JÚNIOR, J. C; NAHAS, M. V; BARROS, M. V. G; LOCH, M. R; OLIVEIRA,

6. E. S. A; DE BEM, M. F. L; LOPES, A. S. Comportamentos de risco à saúde em adolescentes no Sul do Brasil: prevalência e fatores associados. Rev. Panam Salud Publica/Pan Am J Public Health 25(4):344-52, 2009.

7. GUARDA,F. R. B. Frequência de Pratica e Percepção da Intensidade das Atividade Físicas mais Frequentes em Adultos. Fundação de Educação Superior de Olinda. Pernambuco, Brasil. Rev. Pam-Amoz Saúde, 2010 .

8. FERNANDES, Marcela de Melo; ENHA, Daniel Silva Gontijo; BRAGA, Francisco de Assis. Obesidade infantil em crianças da rede pública de ensino: prevalência e consequências para flexibilidade, força explosiva e velocidade. 2012.

9. QUADRANTE, Ana Catarina. Doenças crônicas e o envelhecimento. 2008. Portal do Envelhecimento. Disponível em: www. portaldoenvelhecimento.org.br/acervo/artieop/Geral/ artigo250. Acesso em 05 Abr.2021.

10. PALMA, Alexandre. Exercício Físico e saúde, Sedentarismo e Doença: Epidemia, Causalidade e Moralidade. Revista de Educação Física, UNESP, v.15, n.1, p.185- 191, jan/mar. 2009.

11. Villanueva K, Giles-Corti B, McCormack G. Achieving 10,000 steps: a com-parison of public transport users and drivers in a university setting. Prev Med, 2008; 47(3): 338-41.

12. BRASIL. SaúdeBrasil. Obesidade é porta de entrada para outras doenças; 2017. Disponível em: https: / / saudebrasil.saude.gov.br/ter-peso-saudavel/ obesidade-e-porta-de-entrada-para-outras-doencas. Acesso em: 15 out 2021.

13. GUEDES!!!! GUEDES, D. P; NETO, J. T. M; GERMANO, J. M; LOPES, V; SILVA, A. J. R. M. Aptidão física relacionada à saúde de escolares: programa fitnessgram. Rev. Bras. Med. Esporte. Vol. 18, No 2 - Mar/Abr, 2012.

OBSERVAÇÃO: Os autores declaram não existir conflitos de interesse de qualquer natureza. 